\title{
PERCEPÇÕES SENSORIAIS NAS POSSIBILIDADES DE TRABALHO DE PESSOA COM DEFICIÊNCIA VISUAL: UM ESTUDO DE CASO
}

\author{
Maria Fernanda Berna Lopez VALÉRIO ${ }^{1}$ \\ Fernanda Carla Wasner VASCONCELOS ${ }^{2}$ \\ Daniela Almeida do AMARAL ${ }^{3}$
}

1 Química, Mestre em Turismo e Meio Ambiente, Docente de Graduação do Centro Universitário UNIBH, fernandalopezval@yahoo.com.br

2. Bióloga; Doutora em Ciências; Docente de Graduação e Mestrado do Centro Universitário UNA, fernanda.wasner@prof.una.br

${ }^{3}$.Nutricionista, Mestre em Ciências Biológicas, dani_almeida78@zipmail.com.br

Recebido em: 21/10/2013 - Aprovado em: 20/12/2013 - Disponibilizado em: 15/01/2014

RESUMO: O propósito deste artigo foi analisar comparativamente as possíveis divergências nas percepções entre deficientes visuais e indivíduos videntes na aplicação da técnica de análise sensorial de aromas. Por meio da coleta de dados, foram entrevistados 59 sujeitos (grupo experimental e grupo de controle), com base em questionários semiestruturados. A análise dos dados foi quali-quantitativa. Os resultados mostram que o sistema olfativo tem características únicas. Verificou-se que as percepções quanto aos cheiros são diferentes, cada pessoa estabelece uma relação própria com o aroma que está sentindo, e isso se dá em função das suas experiências pregressas envolvendo aquele cheiro específico. Observou-se que os não videntes são mais atentos à forma como eles percebem cheiros e que usam mais a imagem olfativa, mas não têm o sentido do olfato mais apurado do que os videntes. Comprovou-se também que na aplicação correta da análise sensorial de aromas, uma empresa poderá ter profissionais (analistas sensoriais) treinados para atuar tanto no controle de qualidade quanto no desenvolvimento de um determinado tipo de trabalho. Foi possível constatar que algumas adequações devem ser feitas nos testes de análise sensorial visando suprir as necessidades do grupo experimental. Concluiu-se que "a boa aceitação de uma empresa" está relacionada à criação de um modelo sustentável de desenvolvimento para toda a sociedade e de como está incentivando o trabalho inclusivo, absorvendo as demandas e necessidades de todos os segmentos sociais, promovendo assim oportunidade para todos.

Palavras-chave: Videntes. Deficientes Visuais. Análise Sensorial. Aromas.

\begin{abstract}
The purpose of this article was to analyze the possible differences in perceptions between visually impaired and psychics individuals in applying the technique of sensory analysis of flavors'. Through given collection, we interviewed 59 subjects (experimental group and control group), based on questionnaires. Given analysis was primarily qualitative and quantitative. The results show that the olfactory system has unique characteristics. It was found that perceptions about the smells are different, each person establishes a proper relationship with the scent you're feeling, and this is a function of their past experiences involving that particular smell. It was observed that the visionaries are not more attentive to how they perceive smells and rely more on olfactory image, but they have no sense of smell more accurate than the sighted. It was shown also that the correct application of sensory analysis of flavors, an enterprise may be professionals (analysts sensory) trained to act both in quality control and the development of a particular type of work. It was that some adjustments must be made in the tests of sensory analysis in order to meet the needs of the experimental group. Thus the adequacy sensory analysis may allow better access to the labor market by the blind. It was concluded that "the acceptance of a good company" is related to the creation of a sustainable model of development for society and how the work is encouraging inclusive, absorbing the demands and needs of all segments of society, thus promoting equality all.
\end{abstract}

Keywords: Psychic. Visually Impaired. Sensory Analysis. Flavor.

\section{INTRODUÇÃO}

O propósito deste artigo foi analisar comparativamente as possíveis divergências nas percepções entre deficientes visuais e indivíduos videntes na aplicação da técnica de análise sensorial de aromas.

Para tanto, antecipou-se em considerar que a inserção das pessoas com deficiências visuais no mercado de trabalho modifica-se de 
acordo com cada empresa. Em algumas organizações essa inserção passou a ser vista como um "objeto" que precisa ser adquirido com rapidez, mesmo que muitas vezes, a empresa não tenha as competências necessárias para realizar, com o devido cuidado, sua inclusão. A contratação de uma pessoa com deficiência visual tornou-se uma das "obrigações" perante o Estado, tanto quanto o pagamento de impostos (BRASIL, 1999).

Em decorrência de deficiências sensoriais, algumas pessoas com deficiências visuais apresentam necessidades específicas, caminhos e formas peculiares de apreender e assimilar o real. Necessitam de mais tempo para vivenciar e organizar suas experiências, aprender e construir conhecimentos. Compreendidas essas especificidades pela família, pelos professores e pelas empresas, todos poderão se beneficiar e obter sucesso na inclusão social (BRASIL, 2009).

O que se pretende é considerar que as pessoas com deficiências visuais não são muito diferentes das outras, pois, desde crianças, têm as mesmas necessidades afetivas, físicas, intelectuais, sociais e culturais (AUSUBEL, 1982). Entretanto os Deficientes Visuais (DVs) têm o olfato mais apurado que pessoas videntes, o que é corroborado por alguns autores. Segundo Santin e Simmons (2000), não tendo a visão, os sentidos restantes se adaptam para funcionar sem a segregação que a visão oferece imediatamente. A visão completa os sentidos restantes, e embora sua ausência limite o indivíduo, não o incapacita (CUNHA, 2004).

Destaca-se que há algumas indústrias potenciais empregadoras de deficientes visuais com olfatos apurados: cafés, vinhos, cosméticos e óleos. Portanto, neste estudo, pretende-se avaliar a percepção de deficientes visuais em relação aos aromas. Optou-se pela indústria de óleos essenciais devido ao apoio institucional fornecido pela Lazlo Aromaterapia Ltda, que atualmente projetos de apoio e incentivo à pesquisa das terapias holísticas e complementares, desenvolvimento humano e manejo de agricultura ecos sustentável.

Além dessa organização, a Escola Estadual São Rafael disponibilizou alguns alunos para a pesquisa. Obteve-se a aprovação do Comitê de Ética em Pesquisa do Centro Universitário UNA.

A relevância deste estudo para o meio acadêmico, organizacional e social consiste na ampliação do conhecimento sobre o tratamento que as empresas têm dado à mão de obra com características diferenciadas, a das pessoas com deficiências visuais. Além disso, merece destaque a abordagem das práticas de educação socioambiental aplicadas a esse contingente, visando potencializar seu desempenho e adaptá-lo às condições organizacionais.

Esse compromisso com a realização social de uma cidadania ativa e uma sociedade 
humanizada se expressa de várias formas. Neste trabalho, pretendeu-se comprovar a possibilidade da inclusão social de pessoas com deficiência visual no mercado de trabalho.

Diante do exposto, procurou-se problematizar e delimitar o estudo de maneira que se pudesse investigar quais as semelhanças e diferenças de percepções entre deficientes visuais e indivíduos videntes na aplicação da técnica de análise sensorial de aromas.

\section{INSERÇÃO DE PESSOAS COM DEFICIÊNCIA NO MERCADO DE TRABALHO}

O trabalho é um dos meios para que ocorra a inclusão, pois por intermédio dele o indivíduo, ao se inserir em uma rede de identificação e pertencimentos, relaciona-se e representa o seu mundo, tendo, assim, um processo de simbolização e de reconhecimento social (BATISTA, 2004).

No mercado de trabalho, a inclusão de Pessoa com Deficiência Visual (PcD) é mais do que contratar um deficiente, implica em modificar e questionar padrões preestabelecidos geradores de exclusão. Está relacionada a uma nova forma de se organizar cujo modo é capaz de atender às necessidades de seus membros, principalmente, do portador de deficiência.

Estar empregado no mercado formal, além de permitir ao indivíduo fazer parte de uma organização, traz a ele "o reconhecimento por meio da legalidade e dos processos ideológicos implícitos nesse tipo de contratação" (BATISTA, 2004, p.22). Assim, estar empregado formalmente é um dos caminhos que as pessoas com deficiência percorrem para serem reconhecidas socialmente.

Todavia, a inserção da PcD em determinado ambiente ou determinada empresa, segundo Kauchakje (2000, p.20), não significa que ela está sendo incluída. A inclusão "é o processo de um movimento dinâmico e permanente que reconhece a diversidade humana e tem como fundamento a igualdade na participação e na construção do espaço social, compreendida como direito".

A partir disso, conclui-se que o trabalho, além de fonte de realização e sustento, é um dos caminhos para que as pessoas com deficiência encontrem reconhecimento social. É um dos meios para que ocorra a inclusão, porém, embora surjam oportunidades de trabalho, muitas pessoas com deficiência não estão qualificadas para o cargo, bem como muitas organizações não oferecem suporte necessário para a inserção destas.

Já existem empresas que estão procurando adotar "ações de adequação das condições e práticas de trabalho". Entre elas, podem-se citar a Nestlé e Sasazaki. A Sasazaki que, embora ainda não tenha preenchido o número de cotas reservadas para 
as pessoas com deficiência, vem investindo nessas ações. A Nestlé, além de ter preenchido esse número de cotas, vem adquirindo competitividade ao inserir pessoas com deficiência (JORNAL DIÁRIO, 2012).

3

\section{SOCIOAMBIENTAL DAS empresas}

Nas últimas décadas, vêm aumentando o interesse e o investimento do empresariado brasileiro na área social. Com isso, temas como implementação de projetos sociais comunitários, ética e a Responsabilidade Social (RS) são cada vez mais debatidos.

Para Ashley (2002, p.6), RS pode ser definida como o "compromisso que uma organização deve ter com a sociedade, expresso por meio de atos e atitudes que a afetem positivamente, de modo amplo, ou a alguma comunidade, de modo específico". Assim, numa visão ampla, RS é toda e qualquer ação que tem condição de contribuir para a melhoria da qualidade de vida da sociedade.

De acordo com o Business Social Responsible Institute - BSR (2001), principal entidade mundial na área de RS, reunindo 1.600 empresas, não existe definição unânime aceita para RS, mas, de forma ampla, a expressão se refere às decisões de negócios tomadas com base em valores éticos que incorporam as dimensões legais, o respeito pelas pessoas, comunidades e meio ambiente.
Esse instituto afirma que o conceito de empresa socialmente responsável se aplica àquela que proceda no ambiente de negócios de forma que atinja ou exceda as expectativas éticas, legais e comerciais do ambiente social na qual a empresa esteja engajada.

Nesse sentido, Calixto (2007, p.1) coloca que nas últimas décadas, a preocupação das empresas com investimentos em atividades sociais e ambientais apresentaram crescimento relevante, pois neste mundo globalizado e competitivo elas precisam constituir uma imagem positiva, reforçando sua marca. Podese dizer que o "contexto social e econômico atualmente tem demonstrado a necessidade de inserção da variável socioambiental como preocupação primordial nas decisões empresariais entre países e regiões" (CALIXTO, 2007, p.1), pois a sociedade civil tem condições de se mobilizar para retaliar empresas irresponsáveis que exibem um aspecto negativo na globalização econômica.

Uma empresa socialmente responsável no campo da preservação ambiental destaca por sua excelência em política de gestão ambiental, pela atuação como agente de fomento do desenvolvimento sustentável local e regional. Além disso, pela preservação da saúde, da segurança e da qualidade de vida de seus funcionários e da comunidade, e pela inserção da questão ambiental como valor de sua gestão e como compromisso, sob a forma de missão e visão do seu desempenho 
organizacional (MELO NETO; FROES, 2001).

No momento atual, verifica-se um crescente movimento pela ética nas empresas, principalmente nas que buscam a sua sustentabilidade, uma vez que ela é à base da responsabilidade social e ambiental, expressa nos princípios e valores adotados pela organização.

Ferrel, Fraedrich e Ferrell (2001) salientam que as empresas precisam reagir de maneira ética às necessidades dos stakeholders e de outros membros da sociedade que se mostrem interessados. É importante que elas funcionem de modo compatível com os padrões sociais e normas éticas. Para que a ética seja parte da RS, a estratégia das empresas deve refletir a compreensão dos valores de seus membros e dos stakeholders, e a natureza ética da opção estratégica.

Vê-se, pois que a questão ambiental tem chamado cada vez mais atenção das pessoas, também está presente não somente nos discursos e relatórios divulgados pelas empresas, mas encontram-se inseridos em seus sistemas de gestão, processos e normas internas (CALIXTO, 2007).

\section{METODOLOGIA DA PESQUISA}

Quanto ao tipo de pesquisa, o estudo caracterizou-se como pesquisa de campo adequada a estudos experimentais (MARCONI; LAKATOS, 2006), possibilitando aos participantes o contato com amostras dos diferentes aromas e suas misturas em diferentes intensidades.

A técnica utilizada foi a identificação de aromas, comum no treinamento de equipes julgadoras de produtos específicos, com a finalidade de ambientar o provador com os aromas, para poder julgar com competência técnica. Segundo Vergara (2000), a pesquisa experimental "é a investigação empírica na qual o pesquisador manipula e controla variáveis independentes e observa as variações que tais manipulação e controle produzem em variáveis dependentes".

$\mathrm{O}$ procedimento foi desenvolvido em consonância às normas estabelecidas pelo Comitê de Ética em Pesquisa do Centro Universitário UNA, sendo estabelecidas parcerias com a Escola Estadual São Rafael e os cursos de Nutrição e Enfermagem do Centro Universitário UNA além do Corpo de Bombeiros/MG.

Caracteriza-se como método de estudo, o estudo de caso, pois se trata de tema ainda não explorado e pouco definido no que diz respeito a percepções de pessoas com deficiências visuais.

O estudo foi realizado com dois grupos. Para o primeiro grupo, participaram estudantes universitários de uma instituição de ensino particular, 15 sujeitos adultos de ambos os sexos, com faixa etária entre 18 e 50 anos, com visão preservada e que demonstraram vontade de participar do experimento, 
constituindo-se o grupo controle e os outros 14 indivíduos foram selecionados da escola do Corpo de Bombeiros de Minas Gerais. O grupo experimental foi composto por 30 deficientes visuais de um universo de 513, participantes da Escola Estadual São Rafael, localizada no município de Belo Horizonte/MG, com a mesma faixa etária.

Cada indivíduo foi instruído sobre os objetivos e os procedimentos envolvidos no estudo. Os participantes assinaram o Termo de Consentimento Livre e Esclarecido (TCLE), autorizando a utilização dos dados da pesquisa para publicação. Foi salientado que estes poderiam retirar seu consentimento, a qualquer momento, sem que isso acarretasse qualquer prejuízo para este indivíduo. Foi postulado também que o experimento não envolve nenhum tipo de risco para a saúde humana.

Os indivíduos foram submetidos à anamnese, que consiste em entrevista realizada pela pesquisadora, buscando elencar todos os fatos que se relacionam com a doença, e à pessoa doente, para coleta sistemática de dados e cadastramento no estudo. Durante a entrevista, os indivíduos responderam a um questionário contendo os seus dados pessoais, a eventual presença de patologias, o uso de medicamentos, se tem o hábito de fumar. Para o grupo experimental, foi avaliado ainda o tipo de deficiência visual, presença de outras deficiências e o nível de aprendizagem em Braille. Estes dados foram coletados em entrevistas individualizadas e realizadas pela pesquisadora, utilizando a ficha de coleta de dados.

Os critérios para inclusão dos indivíduos de ambos os grupos foram, não portadores de patologias crônicas e não usuários de medicamentos que pudessem influenciar na percepção sensorial. Após entrevistados, individualmente, os sujeitos aptos foram convidados a participarem do estudo.

Posteriormente, aplicou-se a técnica de análise sensorial, na qual o poder discriminatório para aromas dos julgadores do grupo experimental e do grupo controle foi avaliado através do contato destes com as amostras de diferentes odores e intensidades.

As fases dos procedimentos de metodológicos foram subdivididas em: diluição, preparação, testes sensoriais e coleta de dados, como descrito no Quadro 1.

Quadro 1 - Fases dos procedimentos de metodológicos.

\begin{tabular}{|c|l|}
\hline FASES & \multicolumn{1}{c|}{ DESCRIÇÃO } \\
\hline \multirow{2}{*}{ DILUIÇÃO } & $\begin{array}{l}\text { Após testes primários de diluição, utilizando como base óleo mineral, óleo de milho e óleo } \\
\text { de soja, verificou-se que o óleo de milho era mais adequado para os testes de aroma visto a } \\
\text { relação custo/benefício, por não interferir no aroma principal a ser analisado (essências), por } \\
\text { permitir que a diluição fosse preservada por um maior tempo. Isto permitiu uma maior } \\
\text { veracidade do aroma a ser percebido ao longo do período de teste. }\end{array}$ \\
\hline
\end{tabular}




\begin{tabular}{|c|c|}
\hline PREPARAÇÃO & $\begin{array}{l}\text { As amostras foram preparadas com óleos essenciais, doados pela Laszlo Aromaterapia Ltda. } \\
\text { e diluídos em óleo de milho em diferentes concentrações, de acordo com protocolo } \\
\text { estabelecido previamente. Posteriormente, foram apresentadas aos participantes dos grupos } \\
\text { em copos de vidro, tampados e previamente esterilizados com água fervente para neutralizar } \\
\text { odores. As soluções são de óleo essencial de limão siciliano, gengibre, laranja doce, anis } \\
\text { estrelado, canela da china, cravo botões, café torrado, capim limão, nas concentrações: } \\
\text { muito diluída, diluída, média, concentrada (forte e muito forte). }\end{array}$ \\
\hline $\begin{array}{c}\text { TESTES } \\
\text { SENSORIAIS }\end{array}$ & $\begin{array}{l}\text { Durante os testes sensoriais, feitos em salas separadas, cada julgador foi orientado a } \\
\text { destampar a amostra, aproximar da narina e dar cheiradas curtas, evitando longas inalações } \\
\text { para evitar cansar o olfato pela adaptação. Após a avaliação, o indivíduo foi instruído a } \\
\text { tampar rapidamente a amostra para evitar dissipação do aroma. As soluções a serem } \\
\text { avaliadas a cada encontro foram sorteadas da seguinte maneira: no primeiro encontro, foram } \\
\text { avaliadas as soluções de limão siciliano e canela; no segundo encontro, gengibre e laranja } \\
\text { doce; no terceiro encontro, anis estrelado e cravo botões; e, no quarto encontro, café torrado } \\
\text { e capim limão. A avaliação foi realizada em horários previamente agendados com os grupos } \\
\text { em salas individuais para evitar a comunicação entre os julgadores. Nessas salas, foi } \\
\text { colocada a bandeja contendo amostras de diferentes intensidades codificadas adequadamente } \\
\text { para a discriminação visual ou tátil. }\end{array}$ \\
\hline $\begin{array}{c}\text { COLETA DOS } \\
\text { DADOS }\end{array}$ & $\begin{array}{l}\text { Os dados foram coletados em entrevistas individualizadas realizadas pela pesquisadora. O } \\
\text { avaliador respondeu verbalmente e um pesquisador treinado registrou sua resposta em } \\
\text { formulário próprio, em que foram tabulados e, posteriormente, analisados utilizando análise } \\
\text { quali-quantitativa. As soluções foram avaliadas em escala decrescente de intensidade para } \\
\text { que o poder discriminatório seja construído ao longo do processo. }\end{array}$ \\
\hline
\end{tabular}

Fonte: Dados da pesquisa (2011)

A pesquisa apresentou caráter qualiquantitativo concordando com Paulilo (2007) que somente quando as duas abordagens são utilizadas dentro dos limites de suas especificidades é que podem dar uma contribuição efetiva para o conhecimento.

As análises foram agendadas nos intervalos das refeições, nos horários da manhã ou da tarde, conforme disponibilidade do grupo. Não foram realizados testes em horários que antecedem uma hora após as refeições para evitar alterações da percepção sensorial por fome ou ingestão prévia de alimentos.

O estudo foi submetido e aprovado pelo Código de Ética para Pesquisa em Seres Humanos do Centro Universitário UNA e o Conselho Nacional de Saúde, conforme Resolução n 196/1996. Não foram utilizadas técnicas invasivas, não envolveu nenhum risco para os participantes.

\section{RESULTADOS E DISCUSSÃO}

A caracterização dos 59 participantes foi feita com base nas perguntas do roteiro das fichas de anamnese tanto para o grupo experimental quanto para o grupo de controle e se refere à condição, gênero, ser fumante ou não, uso de medicamento e faixa etária.

No que concerne à condição, 50,8\% dos entrevistados são videntes enquanto 49,2\% são cegos.

Em relação ao gênero, existe uma proporção ligeiramente maior do sexo feminino, compreendendo $55,9 \%$ dos entrevistados. 
Verifica-se que $\quad 96,6 \%$ dos entrevistados não fumam, sendo que apenas $3,4 \%$ dos entrevistados são fumantes.

$\mathrm{Na}$ análise das respostas, constatou-se que $75,6 \%$ dos entrevistados não usam medicamentos para tratar qualquer tipo de doença sendo que $24,4 \%$ os utilizam diariamente.

Pode-se observar que $23,7 \%$ dos entrevistados, encontram-se na faixa etária de 18 a 25 anos, de 26 a 35 anos compreendem $23,8 \%$ e, de 36 a 40 anos, contemplam 18,6\% dos participantes.

Constatou-se que $13,6 \%$ correspondem aos julgadores na faixa etária de 41 a 45 anos e 20,3\% na faixa etária de 46 a 50 anos.

\subsection{Aplicação da técnica de análise sensorial de aromas}

As soluções foram avaliadas por um pequeno grupo de 10 pessoas videntes, alunos de nutrição e alguns funcionários da escola UNA ressaltando-se que uma delas conseguiu perceber quase $90 \%$ das amostras e outra, que estava gripada, não conseguiu identificar a maioria dos aromas, comprovando não ser bom fazer testes com pessoas que tenham algum comprometimento das vias respiratórias.

Grupos de não-videntes em relação as amostras in natura: assim, como os videntes, tinham na maioria conhecimento de todos os aromas, somente alguns não conheciam anis estrelado e gengibre, no geral nenhuma novidade, mas quando as diluições foram apresentadas ficou ainda mais difícil a identificação do aroma.

Os não-videntes mais novos encontraram dificuldades, uma vez que já tinham sentido o cheiro, mas nunca apalparam as amostras ao natural.

Logo, observou-se que o deficiente usual deve ser treinado para criar uma boa memória olfativa o que facilitará a identificação dos diferentes aromas nas intensidades apresentadas, ao serem submetidos às técnicas de análise sensorial para aromas.

\section{CONCLUSÃO}

O presente trabalho evidenciou a aplicação da técnica de análise sensorial de aromas, com base em alguns pressupostos da inserção da PcD visual no mercado de trabalho. A ampliação dessa discussão é pertinente, do ponto de vista da aplicação prática, e oferece subsídios para a política inclusiva.

A questão da análise sensorial do trabalho foi realizada em função das respostas transmitidas pelos videntes e cegos, algumas sensações que se originaram de reações fisiológicas e foram resultantes de alguns estímulos, gerando a identificação dos óleos essenciais em diferentes intensidades. Para 
tanto, houve interação entre videntes/cegos e os óleos essenciais. Sabe-se que o estímulo é promovido pela técnica de análise sensorial e as sensações por efeitos psicológicos que ao serem produzidas, puderam dimensionar a intensidade dos níveis de aromas em relação ao óleo essencial avaliado. Nesta avaliação, videntes e cegos, em uma percepção somatosensorial, utilizaram o sentido do olfato.

Concluiu-se que o sistema olfativo tem características únicas relacionadas com sua memória olfativa bem como todas as percepções somatossensoriais. Verificou-se que as percepções quanto aos cheiros são diferentes, cada pessoa estabelece uma relação própria com o aroma que está sentindo, e isso ocorre em função das suas experiências pregressas envolvendo aquele cheiro específico. Em linhas gerais, observou-se que os não videntes são mais atentos à forma como eles percebem cheiros e que usam mais a imagem olfativa, mas não têm o sentido do olfato mais apurado do que os videntes.

Realça-se que em relação aos aromas, a distribuição da frequência da pesquisa se mostrou relativamente uniforme, com predominância tanto de videntes quanto de cegos que apresentaram os mais diferentes comportamentos na percepção dos aromas estudados em suas intensidades muito fraca, fraca, média e forte. Neste contexto, observouse que os deficientes (cegos) tiveram os menores índices de acerto para gengibre, cravo em botões e limão siciliano. Entretanto, para os videntes, este comportamento foi constatado para os aromas gengibre, café torrado e cravo em botões.

Em linhas gerais, é possível afirmar que os deficientes visuais apresentaram melhor percepção do aroma de gengibre em todas as intensidades quando comparada aos videntes. Entretanto, para os aromas anis estrelado e cravo em botões, houve melhor aproveitamento dos cegos em relação aos videntes em $75,0 \%$ das intensidades apresentadas. Além disso, verificou-se o mesmo comportamento (melhor percepção dos aromas pelos não videntes) em 50,0\% das intensidades apresentadas para os aromas de limão siciliano, café torrado e capim-limão.

O pior desempenho de reconhecimento de um determinado aroma pelos cegos foi constatado na laranja doce e na canela da china cujos maiores índices de acerto foram realizados pelos videntes.

Neste estudo também foi possível observar comportamentos semelhantes de percepção entre videntes e cegos como a laranja doce na intensidade média e capimlimão na intensidade forte cujos índices foram 20,6\%, - 20,0\%: no café torrado e no capimlimão ambos na intensidade fraca cujos valores são $24,1 \%$ e $23,3 \%$ respectivamente.

Ressalta-se que em toda análise sensorial, o pior índice de acertos foi $3,5 \%$ para videntes ao julgarem o aroma cravo em botões na intensidade fraca e $13,0 \%$ para cegos 
ao apreciarem a amostra de canela da china na intensidade muito fraca.

Cabe destacar que a partir da aplicação correta da análise sensorial de aromas, que uma empresa poderá ter profissionais (analistas sensoriais) treinados para atuar tanto no controle de qualidade quanto no desenvolvimento de um determinado tipo de trabalho mesmo em condições de deficiência visual.

Comprovou-se que esta metodologia quando bem adequada pode possibilitar um melhor acesso ao mercado de trabalho pelos deficientes visuais mas é preciso criar inicialmente a memória olfativa prévia para facilitar o processo de análise.

Observou-se que "a boa aceitação de uma empresa" está relacionada à criação de um modelo sustentável de desenvolvimento para toda a sociedade e de como está incentivando o trabalho inclusivo, absorvendo as demandas e necessidades de todos os segmentos sociais, promovendo assim a igualdade de todos.

Assim, pode-se dizer que a educação socioambiental constitui-se uma ferramenta de gestão moderna e significativa para que as diferentes empresas permitam a prática de políticas inclusivas efetivas, em consonância com os princípios de sustentabilidade que permeiam as exigências do competitivo mercado globalizado.

\section{REFERÊNCIAS}

ASHLEY, Patrícia Almeida. Ética e responsabilidade social nos negócios. São Paulo: Saraiva, 2003.

AUSUBEL, David Paul. A aprendizagem significativa: a teoria de David Ausubel. São Paulo: Moraes, 1982.

BATISTA, Cristina Abraches Mota. Inclusão: construção na diversidade. Belo Horizonte: Armazém de Ideias, 2004.

\section{BAGATINI, Vilson. Educação física para deficientes. Porto Alegre: Sagra, 1987.}

BIEDRZYCKI Aline. Aplicação da avaliação sensorial no controle de qualidade em uma indústria de produtos cárneos. Instituto de Ciência e Tecnologia de Alimentos, Curso de Engenharia de Alimentos Universidade Federal do Rio Grande dos Sul, Porto Alegre, 2008.

BRASIL. Decreto ${ }^{\circ} 3.298$, de 20 de dezembro de 1999. Regulamenta a Lei $n^{\circ} 7.853$, de 24 de outubro de 1989, dispõe sobre a Política Nacional para Integração da pessoa portadora de deficiência, consolida as normas de proteção e dá outras providências. Brasília:

Diário Oficial da União (DOU), $20 \mathrm{dez}$. 1999.

CALIXTA, Laura. Responsabilidade socioambiental: pública ou privada? XXXI ENCONTRO DA ASSOCIAÇÃO NACIONAL DOS PROGRAMAS DE PÓSGRADUAÇÃO EM ADMINISTRAÇÃO. Rio de Janeiro. 2006. Anais... Rio de Janeiro, 2006.

CARDOSO, Maria Graças et al. Plantas aromáticas e condimentares. Laboratório de Química Orgânica do Departamento de Química da Universidade Federal de Lavras (UFLA). Lavras, 2007.

CUNHA, Malíria Flávia. A expressão corporal e o deficiente visual. Revista Benjamin

Constant. Rio de Janeiro, ano 10, n. 28, ago. 2004. 
COORDENADORIA NACIONAL PARA

INTEGRAÇÃO DA PESSOA PORTADORA

DE DEFICIÊNCIA. Declaração de

Salamanca e linha de ação sobre

necessidades educacionais especiais.

Brasília: CORDE, 1994

DISCHINGER, Marta; ELY, Vera Helena

Moro Bins. A importância dos processos

perceptivos na cognição de espaços urbanos

para portadores de deficiência visual. IX

CONGRESSO BRASILEIRO DE

ERGONOMIA. Salvador-BA, 1999.

FERREL, O. C.; FRAEDRICH, John;

FERREL, Linda. Ética empresarial: dilemas, tomadas de decisões e casos. Rio de Janeiro:

Reichmann \& Affonso Editores, 2001.

KAUCHAKJE, S. Inclusão: uma perspectiva social e da conquista de direitos. In:

QUEVEDO, Antônio A.F.; OLIVEIRA, José

Raimundo; MANTOAN, Maria Teresa Eglér.

(Org.). Mobilidade, comunicação e

educação: desafios à acessibilidade.

Campinas: Editora WVA, 2000.

MARTİN, Manuel Bueno; BUENO, Salvatore

Toro (Org.). Deficiência visual: aspectos

psicoevolutivos e educativos. (Trad.) Magali

de Lourdes Pedro. São Paulo: Livraria Santos

Editora, 2003.

MELO NETO, Francisco Paulo; FROES, César. Gestão da responsabilidade social corporativa: o caso brasileiro da filantropia tradicional à filantropia de alto rendimento e ao empreendedorismo social. Qualitymark:

Rio de Janeiro, 2001. 